\title{
A Research and Design of the Individual Course Management System Based on Android
}

\author{
Shijie Zhu ${ }^{1}$, Yiyuan Zhang ${ }^{2}$ and Xiaolin $\mathbf{Y i}^{3}$ \\ ${ }^{1}$ School of Computer and Information Technology, Beijing Jiaotong University, Beijng 100044, China \\ ${ }^{2}$ School of Information Management, Beijing Information Science \& Technology University, Beijng 100092, China \\ ${ }^{3}$ College of Computer Science, Beijing University of Technology, Beijng 100124, China \\ 11283027@bjtu.edu.cn, 371976647@qq.com, yixiaolin@bjut.edu.cn
}

\begin{abstract}
Among the current fast-paced life, with the popularity of smart phones, tablet PCs and other mobile devices, people use smart mobile devices frequency is much higher than the computer. Mobile is already an indispensable tool. In order to use mobile phones to help students and teachers study and work well, especially for individual courses to management, this individual course management system was designed based on the Android system. The system test results showed that this system can remind everyone to submit homework, give the exam time and class time, and make teachers and students work and study in the most effective way.

Index Terms - Individual Course Management, Android System, Mobile Web Development
\end{abstract}

\section{Introduction}

With the popularity of smart phones, tablet PCs and other mobile devices, mobile phones have become an indispensable tool for people. In order to use the phone to help teachers and students learning better and provide a convenient to students in the school curriculum, especially for personal curriculum management, the paper studied the mobile application development technology through the methods of literature and experimental verification, the Individual Course Management System Based on Android has been designed and implemented.

\section{Design of the Individual Course Management System}

\subsection{The System Architecture}

Personal curriculum management system is mainly through the electronic information system using a mobile phone system to remind students and teachers in the school curriculum, assignments, exams and other functions. System which focuses on the realization that the system for managing personal curriculum, so the system should have a high degree of customization to meet the individual and secondly to manage the content related to the course, such as the management of the entire operation, the amount of curriculum management as well as finishing and examination information. The system is divided into four sub-systems: (the overall structure of the system is shown in Figure 2-1).

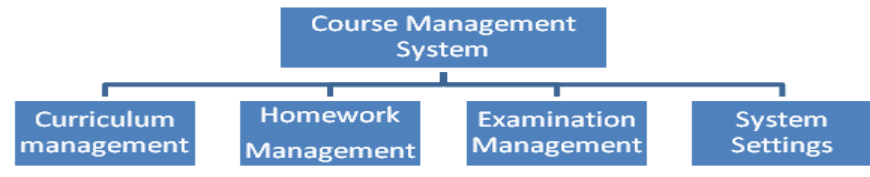

Fig. 2-1 The overall structure diagram of the course management system based on Android system
In Fig. 2-1, the course management subsystem includes three parts of browsing curriculum, adding course and editing course. Browsing curriculum section allows the user to see the distribution of all the courses in a week. Homework management subsystem is divided into four parts of operations, adding homework, editing homework and completing the homework, the main function of which is to complete the job allows users to modify the homework completion status, so that the finished homework will not appear in the reminder list. Exam management subsystem is divided into view, add exam, edit and complete the exam examination of four parts. System settings management subsystem is divided into three parts that include setting semester weeks, setting the reminder time and emptying the database. The subsystem can calculate the current school semester week according to the current date and the start date of the school semester to alert the user, and the subsystem can calculate the alerting items based on the time of the current date, all homework assignments, examinations and the reminder time set by user.

\subsection{The Design Ideas of the System}

Because of the main customers for the system are students and teachers at the school, the application needs should be accessed at any time, and because of the number of users is larger, the information of each person is different, faster data updates, job information changes quickly, the need to regularly update the database data, and most of the teachers and students still use $2 \mathrm{G}$ mode mobile phone, the speed has become a major performance bottleneck for mobile devices. Taking into account the above characteristics, the system is developed by offline single-client mod. In this way, although the signal is weak and slow speed, the teachers and students can also use the system normally to access the course management information about personal curriculum and examination, homework submission time, etc. The above information is directly saved on the user' mobile without any network, it can be simply called from the local database and greatly reduce the cost. Because of the system has no exposure-related data to the outside world interactive interface, it can greatly improved the security of the system.

\section{Research on Key Technologies of the System}

The Individual Course Management System Based on Android includes four sub-systems. This chapter will describe the key technologies in system designing and implementing detailed. 


\subsection{The Key Technology of Building a Database}

The implementation of information input, information query, modification, system settings and other functions, all need the information stored in the database. First a database should be built. The function of create a database is as follows:

Object window. openDatabase (String DBName,

\section{String Version, String Desp , int Space);}

The above function can open the database, and if the database does not exist, it will be created. In which DBName represents the database name, Version represents the version number, Desp represents the depiction, and Space represents the database space. If the database was successfully created, it returns a handle. Then it can use the returned handle (for example, the handle is $\mathrm{db}$ ) to communicate with the database directly. The function of communication is as follows:

db.transaction (function SQLfunction, Function Error)

The above function can transfer SQLfunciton to the database to execute. If an error occurs then call error callback function. SQL function is the database statements, which function method prototype is as follows:

tx.executeSql (String SQLString,

$$
\begin{aligned}
& \text { Array Param, } \\
& \text { Function Success, } \\
& \text { Funtion Error); }
\end{aligned}
$$

This function can be attached to the two functions, corresponding database statement of success and failure corresponding database statement of success and failure callback functions. In which, tx is a variable which passed to SQLfunction automatically by transaction when calling it. The tx includes some function handles of database operations.

\subsection{Android Bottom Interface Selection Method}

Since the system is based on web + native app development of mixed systems, so you need to call the underlying Android interface by JavaScript [1], to achieve at the dawn of time specified by the user prompts to have the upcoming exams and other functions to be submitted .

Here mainly use the Notification API in the Phone Gap [2]. Notification API primarily for visual, auditory and tactile notification of equipment. It consists of four methods: notification. alert, notification. confirm, notification. beep, notification. vibrate. In Android-based personal course management system development, the main methods are notification. alert, notification. beep and notification. vibrate.

Among them, the method notification. alert function is to display a custom warning or dialog function implemented by navigator. notification. alert (message, alert Callback, [title], [button Name]), in which the message is the dialog information, alert Callback is the callback function when the warning dialog is ignored, 'title' is the title of the dialog box , 'button Name' is the button name, notification. beep function is to make the device beep, implemented by navigator. Notification, beep (times) function, which time is the number of beep repetitions times, notification. vibrate function is to make the device vibrate specified length of time, the function implemented by navigator. notification. vibrate (time), which time is the device vibrate time in milliseconds.

\subsection{The Key Technologies of System Alerts}

In individual course management system, we must first get the user's current storage homework and examination information, and organize, identify requirements, such as compliance with the reminder time homework and exam information. The system uses the following methods to achieve:

tx.executeSql ('SELECT * FROM homework ORDER BY time', [],

tt.initHomework['success'], tt.initHomework['error']);

Database can be sorted by time information on all homework listed by the above method, and then we deal with these data: the user set in advance in accordance with homework alerts should be screened for the homework which needs to be submitted in the near future. Others process information data just the same. The following code returns the data according to the database to find a homework unfinished (result.rows.item (i) fin $=1$. !):

Having identified the unfinished homework, get the date (tt.today.getTime ()), plus the time (tt.homework.time * $3600000 * 24)$, to calculate the time when the user need to submit homework. Exam Information is also screened in the same manner, except that test is completed or not, but according to the time set by user and the current time information to determine the test reminder time.

\section{The System Test}

This chapter focuses on the testing and operation of the system, in order to detect the status of the system running.

\subsection{Hardware Environmental Requirements}

The system hardware environment requirements are shown in table 4-1.

Table 4-1 Requirements of Hardware Environment

\begin{tabular}{|c|c|c|}
\hline Category & Recommended & Lowest Configuration \\
\hline Mobile Hardware & CPU 1GHz 1G ROM & $800 \mathrm{MHz} 512 \mathrm{M} \mathrm{ROM}$ \\
\hline Mobile Software & $\begin{array}{c}\text { Android 4.0 Chinese } \\
\text { input method }\end{array}$ & $\begin{array}{c}\text { Android 2.3 Chinese input } \\
\text { method }\end{array}$ \\
\hline
\end{tabular}

\subsection{System Test and Operational Analysis}

4.2.1 Curriculum Management Module

Curriculum management interface used to distinguish 
prominent in seven colors, seven days a week, user-friendly navigation. Click new courses can enter the corresponding interface for the new curriculum operation. Click on the course curriculum can enter the corresponding interface to view course information, as shown in Figure 4-1.

In the new course page, shown in Figure 4-2, after the user enters class time it will automatically verify the current setting of time, if you already have a class it will make a prompt. After you have entered the entire information click on the completion of construction, the information will be recorded in the database and update the curriculum pages of information.

In the course details page, shown in Figure 4-3, it shows the course of the current time information stored in the database and gives two functions: to modify the curriculum and delete courses to choose from.

In modifying the course page, as shown in Figure 4-4, you can modify the course of all information except for class time, after clicking OK modifications, will present data from a database in a timely and prompt modified successfully.

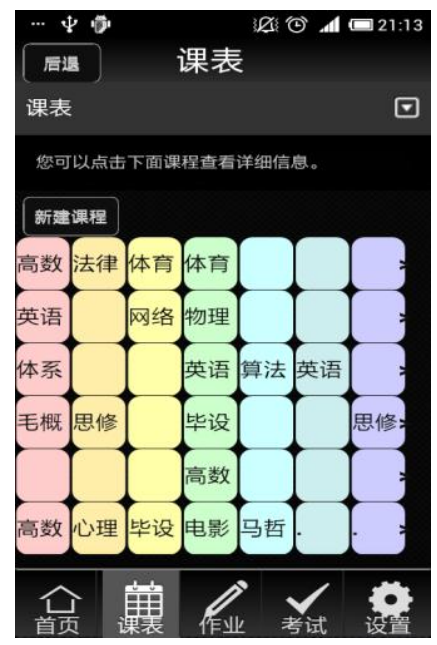

Fig. 4-1 Curriculum management Homepage

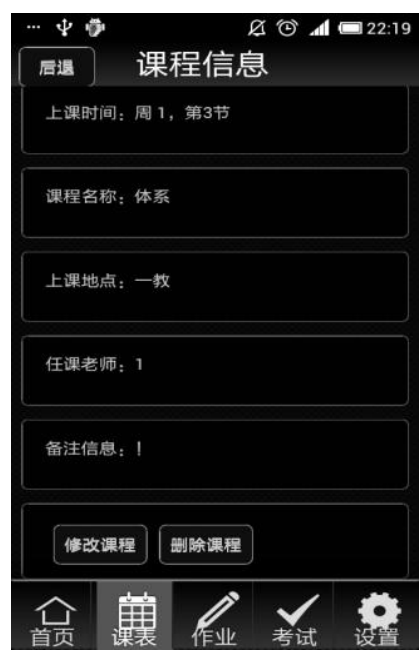

Fig. 4-3 Course Information

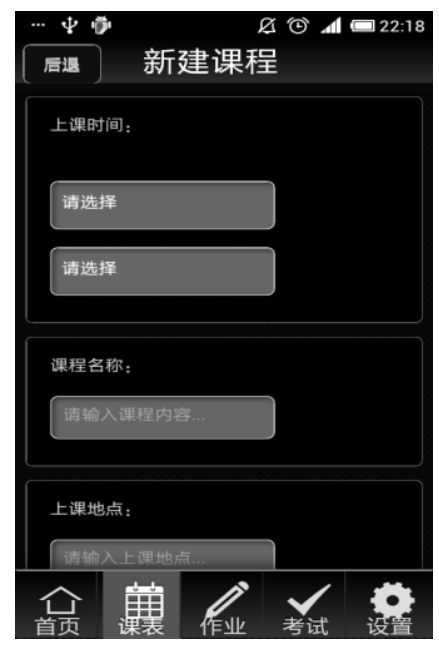

Fig. 4-2 Create Courses

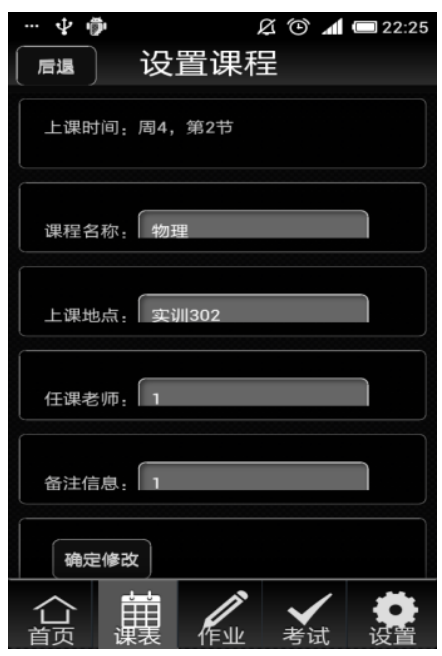

Fig. 4-4 Modify Course

\subsubsection{Homework \& Test Management Module}

As shown in Figure 4-5 and 4-6, homework management module and test management module is basically the same, are the same layout as the first screen showing the current homework and exams, and sorted according to the time before and after. Click Browse all homework / exams can enter the corresponding interface, browse all homework / exams.

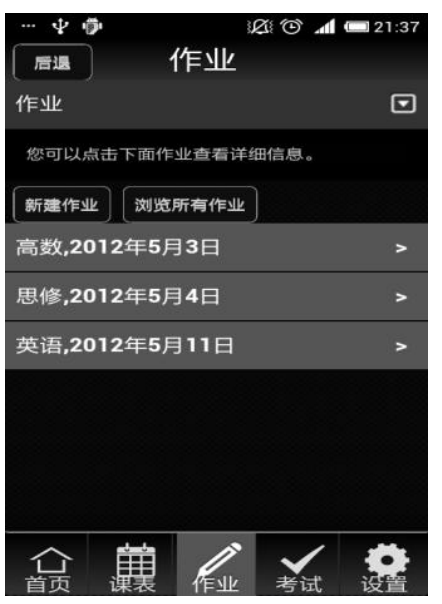

Fig. 4-5 Homework Management Page

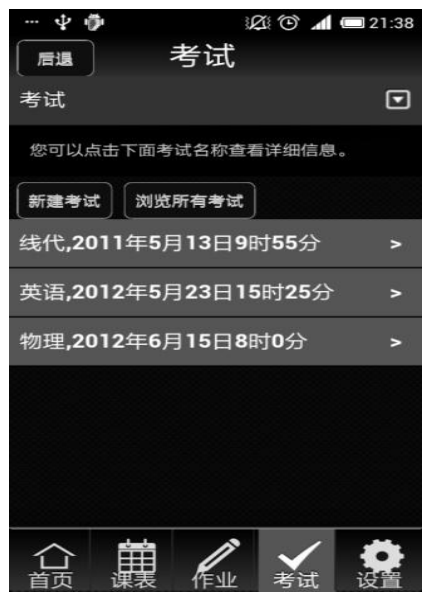

Fig. 4-6 Examination Management Page

\subsubsection{System Settings Module}

As shown in Figure 4-7, in the System Settings page, the operation is simple, you can set the start date, homework alert, the exam questions. Also on this page you can initialize the database, clear all data.

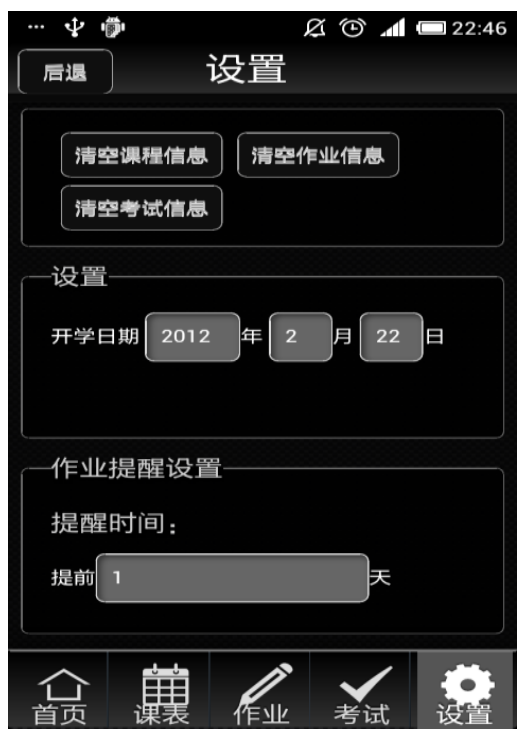

Fig. 4-7 System Settings

\section{Conclusion}

The system provides a convenient query school curriculum function for teachers and students. With mobile phones, users no longer need to take photos of curriculum stored in the phone, but can freely accord to their own needs 
to add course, without network in the system, and can easily record homework and examination. The system can give smart prompt according user's recorded information, so users will not miss any important matter. The Individual Course Management System can truly meet the needs of teachers and students, and provide a function with viable, independent of time, place and network restrictions.

\section{References}

[1] Nicholas C.Zakas. Professional JavaScript for Web Developers 3rd edition. Wrox Press

[2] PhoneGap API Docs http://docs.phonegap.com/en/1.7.0/index.html 